\title{
A Case of Bilateral Medial Posterior Inferior Cerebellar Artery Infarction Associated with Subclavian Steal Syndrome
}

\author{
Hyun-soon Jang ${ }^{1}$, Sook Young Roh ${ }^{1}$, Byung-Su Kim ${ }^{1}$, Young Ik Jung ${ }^{1}$, Jeong Yeon Kim²* \\ 'Department of Neurology, Bundang Jesaeng General Hospital, Seongnam, South Korea \\ ${ }^{2}$ Department of Neurology, Sanggye Paik Hospital, Inje University College of Medicine, Seoul, Korea
}

*Corresponding author: Jeong Yeon Kim, Department of Neurology, Sanggye Paik Hospital, Inje University College of Medicine, Seoul, Korea, E-mail: jykimmd@paik.ac.kr

\begin{abstract}
Subclavian Steal Syndrome (SSS) is the result of reversed ipsilateral vertebral arterial blood flow caused by proximal subclvian artery steno-occlusive disease. A 78-year-old man with a history of hypertension and smoking presented with sudden dizziness, nausea and vomiting; there was no history of recent trauma and arm claudication. Neurologic examination showed no abnormality except for ataxia of both arms. Diffusion Weighted Image (DWI) indicated acute bilateral medial Posterior Inferior Cerebellar Artery (PICA) infarction. Subsequent brain Magnetic Resonance Angiography (MRA) revealed chronic occlusion at the left proximal subclavian artery with decreased left vertebral artery flow and prominent left (PICA) from vertebral artery. Transcranial Doppler (TCD) ultrasonography showed reversal of flow within the left vertebral artery, and reactive hyperemic cuff test of the left arm showed aggravated reverse flow of left vertebral artery following release of blood pressure cuff inflation, which was suggestive of SSS. Although the etiologies of bilateral PICA infarction might be diverse, we experienced a case of acute bilateral medial PICA infarction, which were presumed as hemodynamic insufficiency due to SSS.
\end{abstract}

Received Date: September 1, 2016

Accepted Date: November 6, 2016

Published Date: November 11, 2016

Citation: Kim, J.K., et al. A Case of Bilateral Medial Posterior Inferior Cerebellar Artery Infarction Associated with Subclavian Steal Syndrome. (2016) Int J Neuro Brain Dis 3(4): 1- 3.

DOI: $10.15436 / 2377-1348.16 .1075$

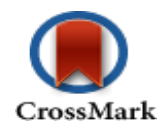

Keywords: Bilateral medical posterior inferior cerebellar artery infarction; Subclavian steal syndrome; Transcranial doppler ultrasonography

\section{Introduction}

Subclavian Steal Syndrome (SSS) is a phenomenon arising from reversed ipsilateral vertebral arterial blood flow caused by proximal subclavian artery steno-occlusive disease. Most cases do not require specific treatment, but some patients show symptoms of arterial insufficiency that affects brain, upper arm and heart, such as paroxysmal dizziness, drop attack and arm claudication ${ }^{[1]}$. Although Transcranial Doppler (TCD) ultrasonography is an useful screening test, the definite diagnosis is made by Computed Tomography (CT) or Magnetic Resonance Angiography (MRA) ${ }^{[2]}$. In asymptomatic SSS, medical therapy is usually recommended, but more invasive methods of intervention or surgical treatment are needed in symptomatic $\mathrm{SSS}^{[3]}$. We experienced a case of bilateral medial Posterior Inferior Cerebellar Artery (PICA) infarction associated with SSS.

\section{Case Report}

A 78-year-old man visited the emergency room with sudden onset of dizziness, nausea and vomiting. He had a history of 30-pack-year smoking and has been on antihypertensive medication. He denied arm claudication and hypo perfusion state such as dehydration, bleeding, burn or shock.

His vital signs were stable, and neurologic examinations revealed alert mentality and normal motor and sensory functions, but weak left radial pulse and ataxia of both upper extremities were observed. Brain Diffusion Weighted Image (DWI) demonstrated acute bilateral medial PICA infarction (Figure 1A). Subsequent MRA revealed occlusion at the left proximal subclavian artery and prominent ipsilatera IPICA from the vertebral artery, however the lumens of both vertebral arteries were intact. (Figure 1B). Manual blood pressure measured

Copyrights: $(0) 2016$ Kim, J.K. This is an Open access article distributed under the terms of Creative Commons Attribution 4.0 International License. 
$153 / 72 \mathrm{mmHg}$ of right arm and 116/71 $\mathrm{mmHg}$ of left arm respectively, with a difference of $37 \mathrm{mmHg}$. Physical examination, laboratory tests, electrocardiography and transthoracic echocardiography revealed normal finding. On the third admission day, TCD showed reversal of flow within the left vertebral artery (Figure 2A). Reactive hyperemic cuff test of the left arm showed aggravated reverse flow of left vertebral artery following release of blood pressure cuff occlusive inflation, which were suggestive of SSS (Figure 2B). His dizziness and ataxia of both arms showed gradually improve don aspirin, clopidogrel and atorvastatin medication. Forty days later, the patient underwent axillo axillary vascular bypass surgery of left subclavian artery. During the follow-up period of 4 months, he was stable with intact left radial pulse
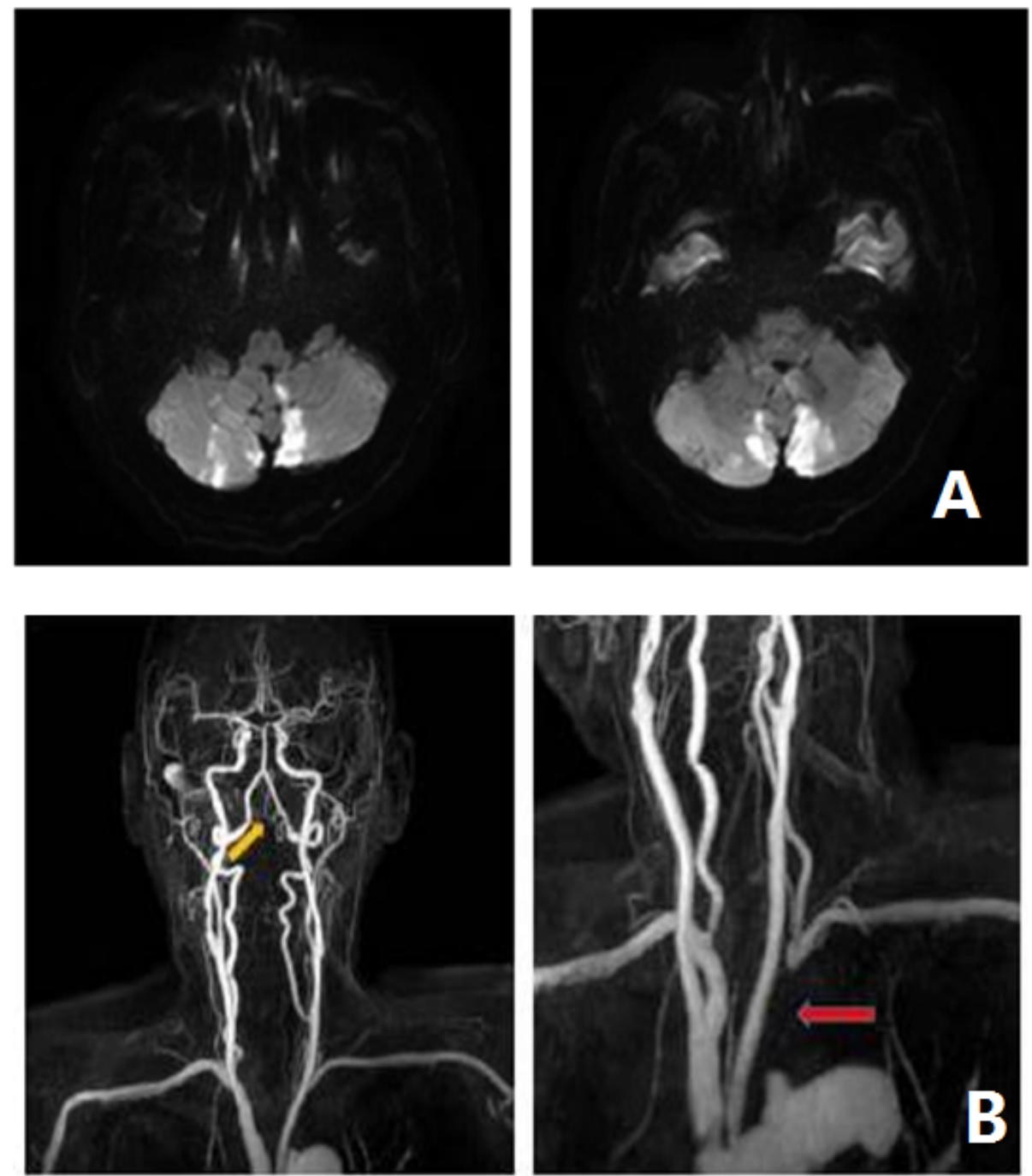

Figure1: Brain diffusion weighted image (DWI) and magnetic resonance angiography (MRA) findings.

(A) DWI showed acute bilateral medial posterior inferior cerebellar artery infarction.

(B) MRA revealed occlusion at the proximal left subclavian artery (red arrow) and prominent ipsilateral posterior inferior cerebellar artery (yellow arrow) from the vertebral artery, however, the lumens of both vertebral arteries were intact.

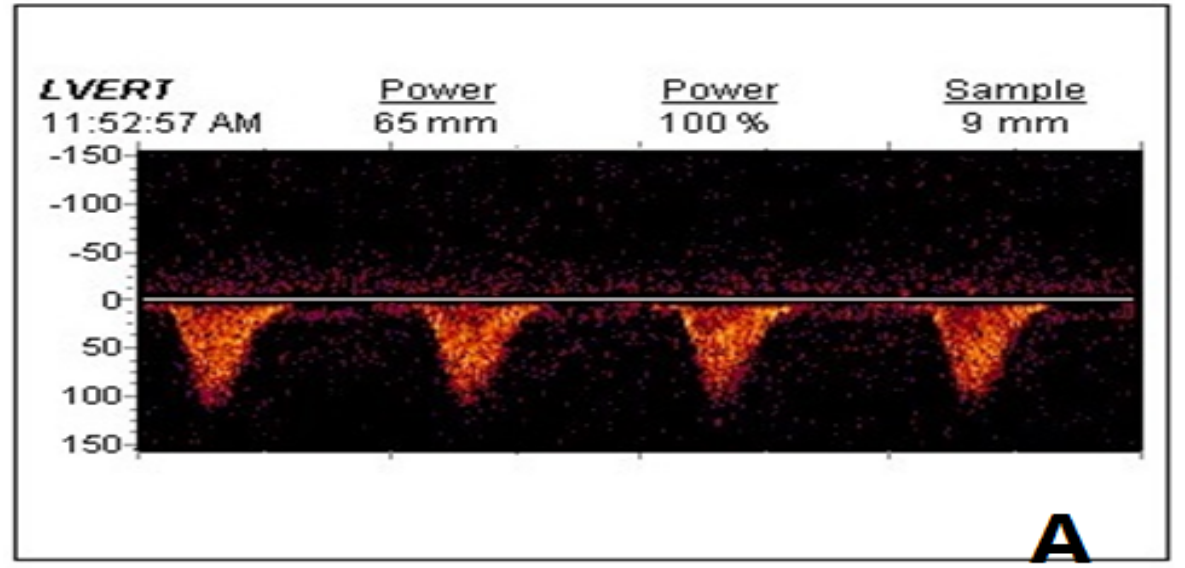




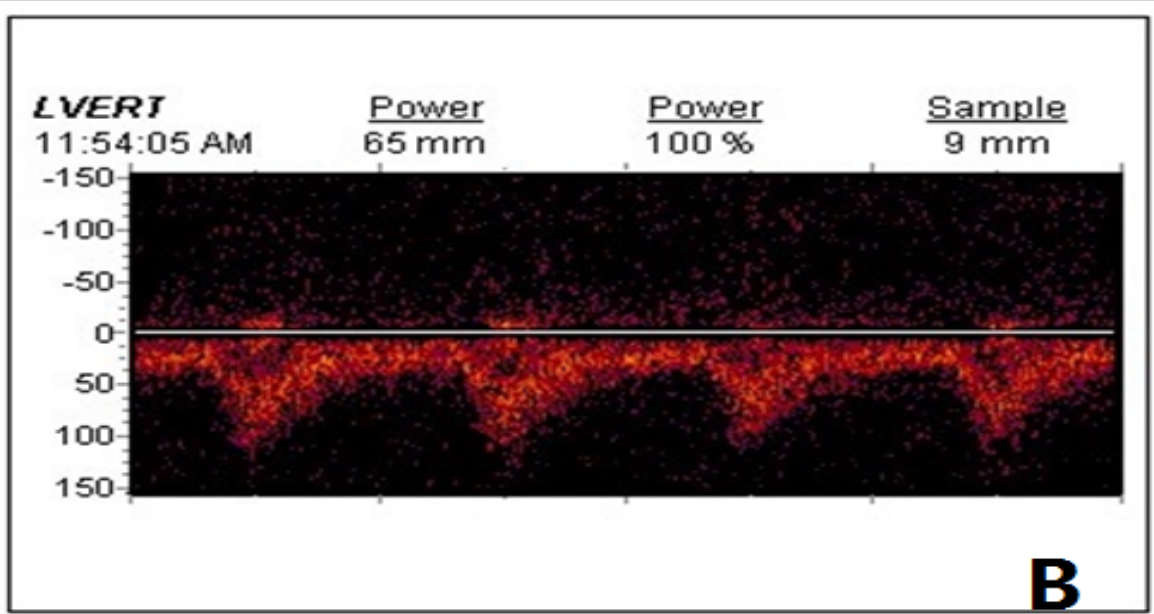

Figure 2: Transcranial Doppler (TCD) ultrasonography and reactive hyperemia test.

(A) TCD showed reversal of flow within the left vertebral artery.

(B) Reactive hyperemia test of the left arm presented aggravated reverse flow of left vertebral artery following release of blood pressure cuff inflation, which was suggestive of subclavian steal syndrome.

\section{Discussion}

SSS is a phenomenon in which ipsilateral vertebral arterial blood flow is reversed due to proximal subclavian artery steno-occlusive disease, which can result in cerebral infarction in rare cases. Although most cases are asymptomatic due to abundant collateral blood supply of head, neck and shoulder area, $5.3 \%$ of SSS patients reportedly present neurologic manifestations ${ }^{[4]}$.

Currently, non-invasive TCD of SSS is accepted as standard bed-side screening test, and has the advantage of evaluation of vertebral arterial hemodynamic and other lesions ${ }^{[5]}$. Furthermore, accurate diagnosis can be made by reactive hyperemia test inducing subclavian steal phenomenon ${ }^{[6]}$.

Our case showed bilateral medial PICA infarction associated with hemodynamic insufficiency due to SSS. Despite absence of vascular abnormalities of posterior circulation on MRA, the finding of bilateral medial PICA territorial lesions is very unusual. PICA is anatomically the most variable blood vessel, and about $20 \%$ of all cases are not visible on angiography, a territory supplied by Anterior Inferior Cerebellar artery (AICA) substitution ${ }^{[7]}$. Brain MRA showed a prominent left PICA alone, however left subclavian artery occlusion induced ipsilateral vertebrobasilar insufficiency even in a single left PICA territory, which finally resulted in acute bilateral medial PICA infarc$\operatorname{tion}^{[8,9]}$.

However, our case had limitations in terms of first clinical presentation of neurologic abnormalities, absence of cerebral angiography findings consistent with cerebellar blood circulation, and high vascular risk factors such as old age, hypertension, and smoking history. These findings highlight that the cause of cerebral infarction can be related not only cerebral ischemia but also other etiologies.

We reported a case of acute bilateral medial PICA infarction associated with SSS, which is an example of diverse patterns of cerebral infarction according to the patient's basic state and variation of blood vessels.

\section{References:}

1. Potter, B.J., Pinto, D.S. Subclavian steal syndrome. (2014) Circulation 129(22): 2320-2323.

2. Osiro, S., Zurada, A., Gielecki, J., et al. A review of subclavian steal syndrome with clinical correlation. (2012) Med SciMonit 18(5): 57-63. 3. Reyna, J., Peguero, J.G., Elmahdy, H.M., et al. Subclavian artery stenosis: a case series and review of the literature. (2014) Rev Cardiovasc Med 15(2): 189-195.

4. Fields, W.S., Lemak, N.A. Joint study of extracranial arterial occlusionVII. Subclavian steal--a review of 168 cases. (1972) JAMA 222(9): 1139-1143.

5. Vecera, J., Vojtísek, P., Varvarovský, I., et al. Noninvasive diagnosis of coronary-subclavian steal: role of the Doppler ultrasound. (2010) Eur J Echocardiogr 11(9): E34.

6. Sharon, M., Asinger, R.W., Hodges, M. Reactive Hyperemia for the Clinical Diagnosisof Subclavian Steal Syndrome: Report of a Case. (1981) Stroke 12(3): 369-371.

7. Kang, D.W., Lee, S.H., Bae, H.J., et al. Acute bilateral cerebellar infarcts in the territory of posterior inferior cerebellar artery. (2000) Neurology 55(4): 582-584.

8. Han, S.W., Cho, G.C., Baik, J.S., et al. Bilateral cerebellar infarction caused by dominant medial posterior inferior cerebellar artery. (2006) Neurology 66(7): 1125-1126.

9. Park, H.Y., Kim, H.J., Cha, M.J., et al. A case of cerebellar infarction caused by acute subclavian thrombus following minor trauma. (2013) Yonsei Med J 54(6): 1538-1541.
Online ISSN: 2377-1348

Journal Title: International Journal Neurology and Brain Disorders Journal Short Name: Int J Neurol Brain Disord
Ommega Online Publishers

E-mail: neurology@ommegaonline.com

Website: www.ommegaonline.org 